\title{
Sugar Metabolism in Yeasts: an Overview of Aerobic and Anaerobic Glucose Catabolism
}

\author{
Fernando Rodrigues, Paula Ludovico and Cecília LeÃo \\ Clinical Mycology Group, Life and Health Sciences Research Institute, Health Sciences \\ School, University of Minho, Campus de Gualtar, 4710-057 Braga, Portugal \\ Fernando Rodrigues (e-mail: frodrigues@ecsaude.uminho.pt) \\ Paula Ludovico (e-mail: pludovico@ecsaude.uminho.pt) \\ Cecília Leão (e-mail: cleao@ecsaude.uminho.pt, Tel.: +351-253-604818, Fax: +351-253- \\ 604809) \\ F.R. and P.L. contributed equally to this chapter.
}

\subsection{Introduction}

Yeasts are ubiquitous unicellular fungi widespread in natural environments colonizing from terrestrial, to aerial to aquatic environments, where the successful colonization is intimately related to their physiological adaptability to a highly variable environment. The metabolic pathways of the central carbon metabolism are basically identical between different yeast species, suggesting that these microorganisms might constitute a metabolic homogenous group. Nonetheless, the mechanisms for nutrient uptake, the number of different isoenzymes and most importantly the regulation of fermentation and respiration differ substantially (Flores et al. 2000) and make yeasts a highly heterogeneous and complex metabolic group.

In yeasts, like other heterotrophic organisms, the energy and carbon metabolism are intimately interconnected, i.e., anabolism is coupled with catabolism. ATP is provided by the oxidation of organic molecules that also act as carbon sources for biosynthesis, and ultimately it is used as energetic currency for all kinds of cellular work.

In the natural environment yeast species have a broad set of carbon sources (e.g., polyols, alcohols, organic acids and amino acids) that can support their growth but preferentially they metabolize sugars. The information related to the metabolism of different carbon sources is huge, the most widely studied being sugars such as hexoses (glucose, fructose, galactose or mannose) and disaccharides (maltose or sucrose) as well as compounds with two carbons (ethanol or acetate). The metabolic networks employed for the metabolism of hexoses and disaccharides share the same pathways (most metabolic building blocks are derived from intermediaries of glycolysis, the tricarboxylic acid cycle (TCA), and the pentose phosphate pathway) and differ only in the initial basic steps of metabolism. However, significant changes could be observed when the metabolism of sugars is compared with that of the 
two-carbon compounds. In this case, the TCA, the pentose phosphate pathway together with gluconeogenesis and the glyoxylate cycle are essential for the provision of anabolic precursors.

Yeast environmental diversity mostly leads to a vast metabolic complexity driven by carbon and the energy available in environmental habitats. It is the scope of this chapter to contribute a comprehensible analysis of yeast metabolism specifically associated with glucose catabolism in Saccharomyces cerevisiae, under both aerobic and anaerobic environments. A brief introduction to glycolysis together with the most relevant effects triggered by oxygen and glucose are presented in order to center the reader in the problems discussed later. Most of our attention is given to the metabolic flux on the pyruvate branch point, with reference to alcoholic fermentation and respiration. As a last issue we address the most pertinent features of anaerobic metabolism, culminating with the hitherto unexplained metabolic requirements for fully anaerobic growth.

\subsection{A Brief Comment on Pasteur, Crabtree and Custer Effects}

Yeasts may be physiologically classified with respect to the type of energy-generating process involved in sugar metabolism, namely non-, facultative- or obligatefermentative (van Dijken and Scheffers 1986). The nonfermentative yeasts have exclusively a respiratory metabolism and are not capable of alcoholic fermentation from glucose (e.g., Rhodotorula glutinis), while the obligate-fermentative yeasts "natural respiratory mutants" - are only capable of metabolizing glucose through alcoholic fermentation (e.g., Candida slooffii $=$ Kazachstania slooffiae). Most of the yeasts identified are facultative-fermentative ones, and depending on the growth conditions, the type and concentration of sugars and/or oxygen availability, may display either a fully respiratory or a fermentative metabolism or even both in a mixed respiratory-fermentative metabolism (e.g., S. cerevisiae or Pichia jadinii - the latter is herein always referred to as C. utilis).

The sugar composition of the media and oxygen availability are the two main environmental conditions that have a strong impact on yeast metabolic physiology. There are three frequently observed effects associated with the type of energy-generating processes involved in sugar metabolism and/or oxygen availability: Pasteur, Crabtree and Custer. The known Kluyver effect is beyond the scope of the metabolic overview of this chapter (see the review in Fukuhara 2003 for more details).

\subsubsection{Pasteur Effect}

In modern terms the Pasteur effect refers to an activation of anaerobic glycolysis in order to meet cellular ATP demands owing to the lower efficiency of ATP production by fermentation compared with respiration. In I86I Pasteur observed that $S$. cerevisiae consume much more glucose in the absence of oxygen than in its presence. However, there are several misinterpretations concerning the results presented by Pasteur that were reviewed in depth by Lagunas (I98 I, I986, and references therein). This author gathered all the information referred to $S$. cerevisiae and showed that the initial descriptions of Pasteur were an artefact due to anaerobic growth impairment 
by the lack of sterols and fatty acids. In fact, $S$. cerevisiae only shows a Pasteur effect under special experimental conditions, specifically at low growth rates (sugar-limiting continuous culturing) and at resting-cell conditions, where a high contribution of respiration to sugar catabolism occurs owing to the loss of fermentative capacity.

\subsubsection{Crabtree Effect}

The Crabtree effect is currently defined as the occurrence of alcoholic fermentation under aerobic conditions (for a review see Pronk et al. I996, and references therein). After the initial descriptions of Crabtree, it was shown that $S$. cerevisiae catabolyzes glucose mainly by a fermentative process, and this effect was presented as the Crabtree effect (Swanson and Clifton I948). From all the theories presented in the literature one should stress that the Crabtree effect has received special attention from several research teams and, even today, a lack of consensus in terms of its definition is observed. De Deken (I966) described the Crabtree effect as "...the phenotypic expression of a regulatory system involved in the synthesis of cytochromes..." Glucose repression became accepted as synonymous of the Crabtree effect until the I 980 s with the emergence of the theory involving an "overflow/limited respiratory capacities" in the branching point of pyruvate metabolism (Kappeli I986). The Crabtree effect was divided into short- and long-term effects, mainly based on continuous culturing studies where under steady-state conditions the growth rate can be experimentally manipulated (Petrik et al. 1983). The short-term effect is defined as the capability of triggering alcoholic fermentation upon the sudden condition of glucose excess, whereas the long-term effect is characterized as the respirationfermentative metabolism observed with batch cultivations or with continuous culturing above critical dilution rates.

\subsubsection{Custer Effect}

The Custer effect is known as the inhibition of alcoholic fermentation by the absence of oxygen. A clear example of the Custer effect is found in yeasts belonging to the Brettanomyces and Dekkera genera that ferment glucose into ethanol and acetic acid under aerobic conditions (Wijsman et al. I984; van Dijken and Scheffers I986). However, upon a shift to an anaerobic condition, fermentation is strongly inhibited. This phenotype can be fully rescued by the reintroduction of oxygen into the culture media or by the addition of $\mathrm{H}^{+}$acceptors such as acetoin (which is reduced to 2,3-butanediol) or other aliphatic carbonyl compounds (Sheffers 1966; Wijsman et al. 1984). From all the previous considerations it is thought that the Custer effect is caused by reductive stress. It seems that yeasts displaying this effect are somehow incapable of closing the redox balance through the production of glycerol or other highly reduced compounds.

\subsection{Glycolytic Metabolic Central Block: a Brief Summary}

After glucose uptake, intracellular glucose is fated to be dissimilated and/or assimilated by metabolic processes (see reviews in Lagunas I993; Boles and Hollenberg 
I997; Forsberg and Ljungdahl 200I; Jeffries and Jin 2004). In this section we will briefly summarize the glycolytic pathway that constitutes the central block of hexose and disaccharide metabolism and that has been extensively revised during the last few years (for reviews see Gancedo and Serrano 1989; Richard 2003; Kruckeberg and Dickinson 2004).

Once inside the cell, glucose is phosphorylated by kinases to glucose 6-phosphate and then isomerized to fructose 6-phosphate, by phosphoglucose isomerase. The next enzyme is phosphofructokinase, which is subject to regulation by several metabolites, and further phosphorilates fructose 6-phosphate to fructose I,6bisphosphate. These steps are the first part of glycolysis that requires energy in the form of ATP.

The subsequently acting enzymes are aldolase, triosephosphate isomerase, glyceraldehyde 3-phosphate dehydrogenase, phosphoglycerate kinase, phosphoglycerate mutase, enolase and pyruvate kinase. The last block of glycolysis leads to pyruvate formation associated with a net production of energy and reducing equivalents.

Essentially the glycolytic pathway is common to all yeast species, the carbon flux regulation being done at the level of the pentose phosphate pathway. Several flux analyses have shown that approximately $50 \%$ of glucose 6-phosphate is metabolized via glycolysis and 30\% via the pentose phosphate pathway in Crabtree negative yeasts. However, about $90 \%$ of the carbon going through the pentose phosphate pathway reentered glycolysis at the level of fructose 6-phosphate or glyceraldehyde 3-phosphate. The distribution of carbon flux between glycolysis and the pentose phosphate pathway seems to play a more important role in glucose dissimilation in Crabtree negative yeasts than in Crabtree positive ones (Bruinenberg et al. I983; González-Siso et al. 2000). Specifically, S. cerevisiae, a Crabtree positive yeast, was shown to have low catabolic fluxes through the pentose phosphate pathway (Blank and Sauer 2004). These observations indicate that the pentose phosphate pathway in Crabtree positive yeasts is predominantly used for NADPH production but not for biomass production or catabolic reactions, as will be discussed later for redox balances.

\subsection{From Pyruvate to Acetyl-Cofactor A}

At the pyruvate (the end product of glycolysis) branching point, pyruvate can follow three different metabolic fates depending on the yeast species and the environmental conditions (Pronk et al. I996). On the other hand, the carbon flux may be distributed between the respiratory and fermentative pathways.

Pyruvate might be directly converted to acetyl-cofactor A (CoA) by the mitochondrial multienzyme complex pyruvate dehydrogenase (PDH) after its transport into the mitochondria by the mitochondrial pyruvate carrier. Alternatively, pyruvate can also be converted to acetyl-CoA in the cytosol via acetaldehyde and to acetate by the so-called PDH-bypass pathway (reviewed in Pronk et al. 1996). The PDHbypass pathway requires the activity of three different enzymes: (I) pyruvate decarboxylase, which converts pyruvate to acetaldehyde; (2) acetaldehyde dehydrogenase (ALD), which converts acetaldehyde to acetate; and (3-) acetyl-CoA synthetase (ACS), which converts acetate to cytosolic acetyl-CoA that can then be transported 
unidirectionally into the mitochondrion via the carnitine acetyltransferase system (Kispal et al. I99I; Pronk et al. I996) (Fig. 6.I). It is still matter of debate if the flux distribution during pyruvate metabolism determines whether the pyruvate flows inward through the PDH complex or through the PDH-bypass pathway and thereby the split between respiration and fermentation (Pronk et al. 1996).

One of the mechanisms underlying the regulation of pyruvate flux through the different routes is the regulation of the enzymes involved and their kinetic properties (Pronk et al. 1996). Compared with cytosolic pyruvate decarboxylase, the mitochondrial PDH complex has a higher affinity for pyruvate and therefore most of the pyruvate will flow through the PDH complex at low glycolytic rates. However, at increasing glucose concentrations, the glycolytic rate will increase and more pyruvate is formed, saturating the PDH bypass and shifting the carbon flux through ethanol production and beginning the fermentation (Fig. 6.I).

In addition to the different enzyme affinities for pyruvate, the enzymatic activities may also play a role in the regulation of pyruvate flux. In $S$. cerevisiae, high glucose concentrations induce an increase of 3-4 times the pyruvate decarboxylase activity and a decrease of acetaldehyde dehydrogenase activity, favoring the alcoholic fermentation. On the other hand, at low glucose concentrations, pyruvate is mainly converted to acetyl-CoA by the PDH complex. From an energetic point of view PDH-bypass is less efficient owing to the consumption of an ATP molecule, which is converted into AMP by the acetyl-CoA synthetase (Steensma 1997).

As already discussed and particularly in the yeast $S$. cerevisiae, the external glucose level controls the switch between respiration and fermentation. Although

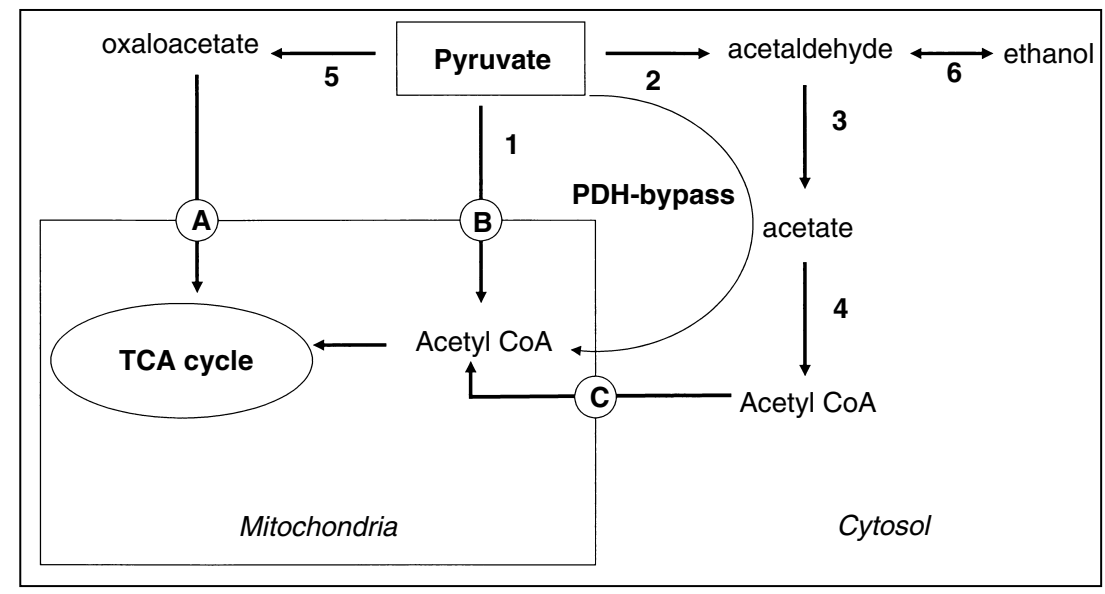

Fig. 6.1. Scheme of pyruvate branch point pathways. Pyruvate formed in glycolysis is converted to acetyl-cofactor $\mathrm{A}(\mathrm{CoA})$ and/or oxaloacetate, both intermediates of the trycarboxylic acid cycle. 1 pyruvate dehydrogenase complex, 2 pyruvate decarboxylase, 3 acetaldehyde dehydrogenase, 4 acetyl-CoA synthetase, 5 pyruvate carboxylase and 6 alcohol dehydrogenase. $A$ mitochondrial oxaloacetate carrier; $B$ mitochondrial pyruvate carrier and $C$ carnitine acetyltransferase 
significant ethanol production is generally absent in Crabtree negative yeasts during aerobic conditions (de Deken I966; González-Siso et al. I996), yeast species such as Pichia anomala, C utilis and Kluyveromyces lactis, during aerobic conditions and high glucose concentration, temporarily (during the exponential growth phase) produce ethanol. However, it has been shown that this transient ethanol production is mainly due to inadequate aeration (Kiers et al. 1998).

\subsection{Tricarboxylic Acid Pathway}

Acetyl-CoA generated either by the PDH complex or by PDH bypass is the link between glycolysis and the tricarboxylic acid (TCA) cycle. The main catalytic function of the TCA cycle is to provide reducing equivalents to the respiratory chain through the oxidative decarboxylation of acetyl-CoA. However, the TCA cycle also functions in furnishing biosynthetic metabolism and, with the exception of isocitrate, every TCA cycle intermediate is commonly used by other metabolic reactions. The eight enzymes from the TCA cycle are encoded by I 5 nuclear genes in S. cerevisiae (McAlister-Henn and Small i997; Przybyla-Zawislak et al. I999). The first reaction of TCA is catalyzed by citrate synthase (encoded by CIT1, CIT2 and CIT3) and it is the condensation of acetyl-CoA and oxaloacetate resulting in the formation of citrate (McAlister-Henn and Small I997). Only Ct2p is a nonmitochondrial protein peroxisomally localized contributing to the efficiency by which the cells use two-carbon compounds in anaplerotic pathways especially in the glyoxylate cycle.

The second reaction of the TCA cycle is catalyzed by aconitase, leading to the conversion of citrate into isocitrate. Aconitase (encoded by $\mathrm{ACOI}$ ) is located both in mitochondria and in cytosol.

The next step of the TCA cycle is the oxidative decarboxylation of isocitrate to $\alpha$-ketoglutarate. There are three known isoenzymes of isocitrate dehydrogenase, a mitochondrial $\mathrm{NAD}^{+}$-specific one and two $\mathrm{NADP}^{+}$-dependent ones (one mitochondrial and the other cytosolic). A number of pieces of evidence point to the role of mitochondrial $\mathrm{NAD}^{+}$-specific isocitrate dehydrogenase in the regulation of the rate of mitochondrial assembly besides its specific role in the TCA cycle (Kruckeberg and Dickinson 2004).

The formation of succinate is catalyzed by $\alpha$-ketoglutarate dehydrogenase, which promotes the oxidative decarboxylation of $\alpha$-ketoglutarate via succinil-CoA to succinate, which is then converted to fumarate by succinate dehydrogenase. The next step of the TCA cycle is the conversion of fumarate to malate by the enzyme fumarase, which exists as separate cytosolic and mitochondrial forms. There is not yet a clear explanation for the existence of these two forms; however, the localization and distribution of fumarase appears to be unique because there is only one translation product which is targeted to mitochondria (Sass et al. 200 I).

Malate dehydrogenase catalyzes the last step of the TCA cycle and leads to the oxidation of malate to oxaloacetate. There are three isoenzymes of malate dehydrogenase: a cytosolic, a mitochondrial and a peroxissomal one; however, the mitochondrial one accounts for $90 \%$ of malate dehydrogenase activity when glucose is being metabolized (Steffan and McAlister-Henn I992). 
TCA cycle flux appears to be constricted at two steps on the basis of the limited availability of the substrates oxaloacetate and $\alpha$-ketoglutarate. As represented in Fig. 6. I, in S. cerevisiae, the synthesis of oxaloacetate from cytosolic pyruvate catalyzed by pyruvate carboxylase constitutes the anaplerotic pathway for the replenishment of this TCA-cycle intermediate. The TCA cycle works in a two-minicycles model interconnecting these two substrates (oxaloacetate and $\alpha$-ketoglutarate) and their transamination products (glutamate and aspartate) (Yudkoff et al. I994; Rustin et al. 1997). This model is consistent with the unique regulation of the first three enzymes of the TCA cycle in yeasts (McCammon et al. 2003).

The genes encoding TCA cycle proteins might also be regulated by glucose levels. In $S$. cerevisiae the depletion of glucose increases $3^{-10}$ times the TCA messenger RNAs (DeRisi et al. I997). Oxygen limitation could also induce a shift in the TCA pathway, which operates as a cycle during aerobic growth and as a two-branched pathway under oxygen limitation, sustaining only the synthesis of the biomass precursor's oxaloacetate and $\alpha$-ketoglutarate as will be discussed hereafter. However, TCA operating as a two-branched pathway was also identified in S. cerevisiae during aerobic fermentation on glucose (Gombert et al. 200 I).

\subsection{Aerobic Metabolism: Oxidative Phosphorilation and Redox Balance}

\subsubsection{Oxidative Phosphorilation}

The mitochondrial oxidative phosphorylation is a complex and highly controlled network through which ATP synthesis must be continuously adapted to changes in the cell energy demand to sustain growth and/or homeostasis. During respiratory metabolism, both cytosolic and mitochondrial NADH are reoxidized by the respiratory chain. However, S. cerevisiae, in contrast to many eukaryotic cells including other yeast species, lacks the multi-sub-unit complex-I-type NADH dehydrogenase (Nosek and Fukuhara 1994). Instead, S. cerevisiae contains a single-subunit NADH:ubiquinone oxidoreductase, which couples the oxidation of intramitochondrial NADH to the respiratory chain. This enzyme (encoded by NDI1), referred to as the "internal NADH dehydrogenase," catalyzes the transfer of two electrons from intramitochondrial NADH to ubiquinone (de Vries and Grivell I988; Marres et al. I99I).

Nonetheless, yeast mitochondria, like those of plants (Moller et al. I993), not only contain the internal mitochondrial NADH dehydrogenase, but also a mitochondrial external NADH dehydrogenase activity (von Jagow and Klingenberg I970). S. cerevisiae has two genes encoding external NADH dehydrogenase isoenzymes, $N D E 1$ and $N D E 2$, both of them typical aerobic expressed genes (Luttik et al. I998; Small and McAlister-Henn I998). Like the internal NADH dehydrogenase, the external isoenzymes do not pump protons (von Jagow and Klingenberg I970). Therefore, S. cerevisiae has a low ATP stoichiometry of oxidative phosphorylation. Notwithstanding this low stoichiometry of oxidative phosphorylation, complete respiratory dissimilation of a glucose molecule yields approximately i6 ATP molecules (four ATP molecules from substrate-level phosphorylation - two from glycolysis and 
two from GTP formed in the TCA cycle - and about I 2 ATP molecules from oxidative phosphorylation). This is eightfold higher than the maximum ATP yield from glucose dissimilation via alcoholic fermentation. The much higher ATP yield from respiratory sugar dissimilation is reflected in the biomass yields of sugarlimited cultures: the typical biomass yield on glucose of respiring cell cultures is fivefold higher than that obtained in fermenting cell cultures (Verduyn i99I).

Another peculiar feature of the $S$. cerevisiae respiratory chain is that the complexes bc I (III) and cytochrome c oxidase (IV) were shown to assemble into large supercomplexes (Schagger and Pfeiffer 2000). The respiratory chain of S. cerevisiae differs from that of other fungi and of plants not only in the presence of the NADH dehydrogenase not coupled to the proton pump but also owing to the absence of a cyanide-insensitive alternative oxidase which catalyzes the direct oxidation of ubiquinone by molecular oxygen without generating a proton motive force (Vanlerberghe and McIntosh I997).

Resting cells of the yeast $S$. cerevisiae have shown that the overall rate of oxidative phosphorylation is short-term-controlled (I) downstream from the ATP synthase by the cytosolic ATP turnover, (2) upstream from the respiratory chain by reducing equivalent availability and allosteric activation of dehydrogenases and (3) by the ionic permeability of the inner mitochondrial membrane (Beauvoit et al. 1993).

Dejean et al. (2000) have shown that mitochondria are the major energy dissipative system in a fully aerobic metabolism and that energy dissipation can be regulated by the decrease in mitochondrial enzyme content to maintain the oxidative phosphorylation regime.

\subsubsection{Redox Balances}

The pyridine-nucleotide cofactors $\mathrm{NAD}^{+} / \mathrm{NADH}$ and $\mathrm{NADP}^{+} / \mathrm{NADPH}$ play a central role in yeast metabolism. NADH is preferentially used in dissimilatory metabolism, whereas NADPH is generally required for assimilatory reactions (van Dijken and Scheffers I986). In S. cerevisiae, C. utilis, and probably in the yeasts in general, NADH and NADPH cannot be interconverted owing to the absence of a transhydrogenase activity (Bruinenberg et al. 1983). The maintenance of a redox balance is a prerequisite for living cells in order to sustain the regular metabolic activity and enable growth. Hence, since biological membranes are impermeable to pyridine nucleotides, to maintain the redox balances, the reduced coenzymes must be reoxidized in the compartment in which they are produced.

Cytosolic NADPH is produced by the oxidative part of the pentose phosphate pathway, which branches off from glycolysis at the level of glucose 6-phosphate. Also, $\mathrm{NADP}^{+}$-dependent isocitrate dehydrogenase and $\mathrm{NADP}^{+}$-dependent acetaldehyde dehydrogenase can contribute to NADPH production (Bruinenberg et al. I983; Minard et al. 1998). S. cerevisiae cannot directly couple the oxidation of NADPH to the respiratory chain (Bruinenberg I986) and therefore is unable to directly oxidize the surplus of cytosolic NADPH. Consequently, the pentose phosphate pathway, which produces the NADPH required for biosynthesis, cannot function as a dissimilatory route in $S$. cerevisiae (González-Siso et al. I996). Also in S. cerevisiae fermentative sugar dissimilation, the role of NADPH is limited, as the major alcohol 
dehydrogenases are strictly NAD ${ }^{+}$-dependent (Ciriacy I979). In contrast, K. lactis uses the pentose phosphate pathway for glucose dissimilation when glycolysis is blocked, suggesting that, in this yeast species, oxidation of the cytosolic NADPH generated in the pentose phosphate pathway can be efficiently coupled to the mitochondrial respiratory chain (González-Siso et al. I996). On the other hand, mitochondrial NADPH dehydrogenases, which couple the oxidation of cytosolic NADPH to the mitochondrial respiratory chain, are common in plants, and this mitochondrial NADPH oxidizing activity has also been reported in C. utilis (Bruinenberg 1986; van Urk et al. 1989) but it is absent from S. cerevisiae (de Vries and Marres I987; van Urk et al. I989; Small and McAlister-Henn I998).

S. cerevisiae has several mechanisms to reoxidize NADH allowing the metabolism to proceed (Bakker et al. 200I). The reduction of $\mathrm{NAD}^{+}$occurs both in the cytosol by glycolysis and in the mitochondria by the PDH complex and dehydrogenases of the TCA cycle. Both pools of NADH can be oxidized by the mitochondrial respiratory chain with oxygen as the terminal electron acceptor (de Vries and Marres I987; Luttik et al. I998; Overkamp et al. 2000; Bakker et al. 200 I).

Aerobically, several systems for conveying excess cytosolic NADH to the mitochondrial electron transport chain exist in S. cerevisiae (Bakker et al. 200 I). The two most important systems in this respect seem to be the external NADH dehydrogenase (Nde Ip/Nde2p) (Luttik et al. I998; Small and McAlister-Henn I998) and the glycerol 3-phosphate shuttle (Larsson et al. I998) although other shuttles could in particular situations play some role (Bakker et al. 200I) (Fig. 6.2). While the external NADH dehydrogenase is suggested as the main system employed for oxidation of cytosolic NADH, the glycerol 3-phosphate shuttle is proposed to be of some importance at low growth rates and perhaps its real significance is only expressed during starvation conditions. The relative importance of these two systems under different conditions is still to a large extent an unsolved matter. Since the external NADH dehydrogenase and the glycerol 3-phosphate shuttle fulfill the same physiological function some kind of regulatory interactions between the two systems would be expected. However, it was shown that the glycerol 3-phosphate shuttle system, which involves the flavin adenine dinucleotide (FAD) dependent Gut2p (Pahlman et al. 200I), is a more efficient system used under conditions where the availability of energy is limited and in fact this system has a higher ATP-to-oxygen ratio compared with the external NADH dehydrogenase (Larsson et al. I998). On the other hand, the external NADH dehydrogenase is superior in terms of producing ATP at a high rate and it is therefore the preferred alternative under most other conditions. Nonetheless, all known pathways of respiratory NADH oxidation in $S$. cerevisiae converge at the ubiquinone pool of the respiratory chain.

Kinetic interactions between NdeIp/Nde2p and Gut2p have been demonstrated since the deletion of either of the external dehydrogenases causes an increase in the efficiency of the remaining enzyme (Pahlman et al. 200I). Moreover, the same authors showed that the activation of NADH dehydrogenase inhibited Gut2p, although this inhibition was not a consequence of the direct action of NADH on Gut2p (Pahlman et al. 200I). Most interestingly is the recent work of Bunoust et al. (2005) where they described that electrons coming from Nde Ip have the right of way over those coming from either Gut2p or NdiI p. Therefore, they proposed that the 


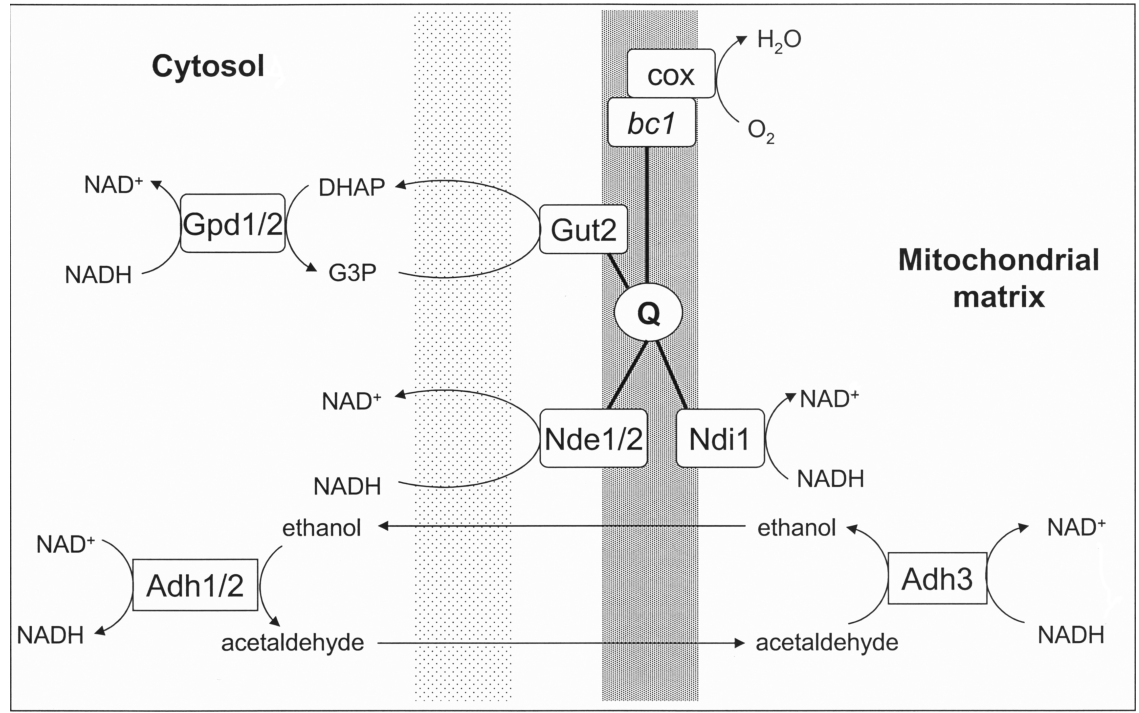

Fig. 6.2. Scheme of redox balance systems in Saccharomyces cerevisiae. Adh alcohol dehydrogenase; $b c l$ bcI complex; cox cytochrome c oxidase; Gpd soluble glycerol 3-phosphate dehydrogenase; Gut2 membrane-bound glycerol 3-phosphate dehydrogenase; Nde external NADH dehydrogenase; Ndil internal NADH dehydrogenase; $Q$ ubiquinone (Adapted from Bakker et al. 200I)

metabolic organization of the respiratory chain is such that it allows a selection and a priority in electron supply, pointing to a new mechanism of regulation of the yeast oxidative metabolism.

The regulation between the two NADH reoxidation systems has also to contemplate the glucose catabolic repression especially when we are talking about $S$. cerevisiae. At high glucose concentrations respiration is only partly repressed (Blomberg et al. I988; Larsson et al. 1998). These traits will render the glycerol 3-phosphate shuttle inactive since one of its components, Gut2p, is subject to glucose repression (Sprague and Cronan 1977). To a large extent, under these conditions, cytosolic redox balance is restored by ethanol and glycerol formation, though the activity of the external NADH dehydrogenase might explain the lower glycerol formation obtained during aerobic compared with anaerobic batch cultures of $S$. cerevisiae (Rigoulet et al. 2004). On the other hand, during aerobic growth at low glucose concentrations in chemostat cultures, both the external NADH dehydrogenase and the glycerol 3-phosphate shuttle seem operative simultaneously (Rigoulet et al. 2004) (Fig. 6.2). However, the NADH dehydrogenase activity appears more important under these conditions.

\subsection{Anaerobic Metabolism}

As referred to before, yeasts may be physiologically classified with respect to the type of energy-generating process involved in sugar metabolism, namely non-, 
facultative- or obligate-fermentative. Within these classes only the last two have a fully operative pathway that can provide free-energy-transduction under anaerobic growth. Alcoholic fermentation is acknowledged as the catabolism of glucose to ethanol. According to the stoichiometry of alcoholic fermentation two molecules of ATP are produced per molecule of glucose converted into ethanol functioning as the main energy supply for maintenance and growth. Additionally, the alcoholic fermentation is a redox-neutral process, since the NADH produced during the oxidation of glyceraldehyde 3-phosphate by glyceraldehyde 3-phosphate dehydrogenase is subsequently reoxidized in the reduction of acetaldehyde to ethanol by alcohol dehydrogenase (van Dijken and Scheffers I986) (Fig. 6.2). However, one cannot overlook that growth is associated with anabolic processes and yeasts have an overall biomass composition that is more oxidized than that of compounds like glucose, which implies that anabolic processes lead to a surplus of reducing equivalents. In contrast to aerobic conditions, the absence of oxygen abolishes the possible oxidation of reduced pyridine nucleotides in the respiratory chain coupled to oxidative phosphorilation. One common way to satisfy the redox balances during growth under anaerobic conditions is the split of glucose metabolism towards glycerol. Glycerol is produced by reduction of the glycolytic intermediate dihydroxyacetone phosphate to glycerol 3-phosphate followed by a dephosphorylation of glycerol 3-phosphate to glycerol. The first step is catalyzed by $\mathrm{NAD}^{+}$-dependent glycerol 3-phosphate dehydrogenase (encoded by the two isogenes GPD1 and GPD2), whereas the second reaction is catalyzed by the activity of glycerol 3-phosphatase (encoded by GPP1 and GPP2) (Eriksson et al. 1995; Larsson et al. 1993; Norbeck et al. I996).

Additionally, the up-regulation of GPD2 associated with an anaerobic growth impairment of $S$. cerevisiae gpd2 mutant clearly establishes the metabolic relevance of this isoenzyme (Ansell et al. 1997; Bjorkqvist et al. I997; Nissen et al. 2000). In the same line of thought, whereas the Gpp2p seems not to play a relevant role in glycerol production under anaerobic conditions, mutants lacking GPP1 display a growth defect under identical conditions. One should stress that the anaerobic growth defects presented by the latter mutant are not due to redox balance impairments but are mainly due to growth-inhibiting levels of intracellular glycerol 3-phosphate (Pahlman et al. 200I). Moreover, anaerobic conditions do not affect expression of GPP2, while GPP1 is induced (ter Linde et al. I999). Hence, the glycerol biosynthesis has defined physiological roles in the metabolic adaptation of $S$. cerevisiae. The correlation between glycerol production and growth under anaerobic conditions is clearly illustrated by the restoring of anaerobic growth defects of mutants with impaired glycerol production by the presence of the electron acceptors acetoin (3-hydroxy-2-butanone) and acetaldehyde. These two compounds alleviate the imposed cellular reductive stress since they enzymatically oxidize intracellular $\mathrm{NADH}$ to $\mathrm{NAD}^{+}$. Furthermore, the increasing flux towards glycerol biosynthesis with the increasing of growth rate is well established. This metabolic shift is the result of an increasing drain from the catabolic pathways to support the higher level of biosynthetic molecules such as RNA and proteins (Nissen et al. 1997).

On the other hand, several yeast species, including $S$. cerevisiae, are well known by their capability of producing acetic acid under both aerobic and anaerobic/ 
oxygen-limiting conditions. In $S$. cerevisiae, the further metabolism of acetic acid through acetyl-CoA synthetase (encoded by $A C S 1$ and $A C S 2$ genes) is the only source of cytosolic acetyl-CoA, an imperative building block of fatty acid biosynthesis (van den Berg and Steensma 1995; Flikweert et al. 1996). When acetaldehyde is converted into acetic acid a surplus of two reducing equivalents is produced. The formation of such a product poses an additional problem in the redox balances, once again circumvented by the production of glycerol. However, if the yeasts cannot cope with the surplus of reducing equivalents the anaerobic activity will come to a standstill, as discussed hereafter. From an anabolic point of view, when acetaldehyde is converted into acetate, NADPH is generated. From the five currently known isogenes of acetaldehyde dehydrogenases, only $A L D 4, A L D 5$ and $A L D 6$ gene products have a role in acetate production during glucose fermentation under both aerobic and anaerobic/oxygen limiting conditions (Remize et al. 2000; Saint-Prix et al. 2004). While the last one encodes a cytoplasmic isoform of acetaldehyde dehydrogenase, the first two encode mitochondrial proteins (Meaden et al. 1997; Tessier et al. I998; Wang et al. 1998). Moreover, this reaction together with the ones catalyzed by malic enzyme (encoded by $M A E 1$ ) and $\mathrm{NADP}^{+}$-dependent isocitrate dehydrogenase (encoded by $I D P I$ ) are currently known to generate mitochondrial NADPH, necessary for biosynthetic purposes.

TCA pathway activity is maintained during fermentation for primary fuel biosynthetic reactions supplying cells with four and five carbon compounds, namely oxaloacetate and 2-oxoglutarate, the precursors of aspartate and glutamate. The TCA pathway is known to operate in a branched fashion under anaerobiosis or conditions of glucose repression (Gombert et al. 200I) where succinate dehydrogenase is nonfunctional (Camarasa et al. 2003). Hence, the flux from 2-oxoglutarate to oxaloacetate is zero. Therefore, the split of the carbon flux in the TCA cycle results in the formation of one oxidative branch leading to the formation of 2-oxoglutarate (Nunez de Castro et al. 1970) and one reductive branch that culminates with the formation of fumarate (Atzpodien et al. 1968). The key enzyme of the redutive branch is the fumarate reductase encoded by FRDS and OSM1 genes (Arikawa et al. I998). The relevant role of this reaction in the reoxidation of intracellular NADH is highlighted by the inability to grow under anaerobic conditions of fumarate reductase null mutant that is restored by the addition of the oxidized form of methylene blue or phenazine methosulfate, which nonenzymatically oxidizes cellular NADH to $\mathrm{NAD}^{+}$(Enomoto et al. 2002). It may perhaps be considered that the role of the enzyme pyruvate carboxylase is to catalyze the reaction of pyruvate to oxaloacetate at the expenses of one molecule of ATP and carbon dioxide (de Jong-Gubbels et al. I998). This anaplerotic reaction functions to further replenish the TCA pathway for biosynthetic purposes or organic acid production as referred to previously.

The synthesis of precursors for biomass building blocks actively results in the net formation of NADH in the mitochondrial matrix (Visser et al. 1994). The reductive branch, thermodynamically favorable, and the $A D H 1 / A D H 3$ shuttle (Fig. 6.2) are two feasible routes for the oxidation of the surplus of NADH produced in mitochondria by both the oxidative TCA branch and the synthesis of amino acids. It should be noted that, stoichiometrically, the formation of succinate by the reductive pathway or by the oxidative pathway results, respectively, in two molecules of FAD 
oxidized or in five molecules of reduced NADH per molecule of glucose consumed. The surplus of reducing equivalents may be reoxidized by the ADH1/ADH3 shuttle involving the mitochondrial alcohol dehydrogenase, adh3p, that results in a net production of NADH in the cytosol and that can be circumvallated by glycerol production (Nissen et al. I997; Bakker et al. 2000). In fact, Nissen et al. (I997) showed that the $A D H 1 / A D H 3$ shuttle is responsible for the regeneration of $\mathrm{NAD}^{+}$, whereas the reductive branch is associated with succinate production. Nevertheless, the $A D H 1 / A D H 3$ shuttle does not strictly control the redox balance under anaerobic conditions, since a null $A D H 3$ mutant still shows anaerobic growth, albeit slower than that of the wild type (Bakker et al. 2000). The TCA pathway during fermentation also leads to the formation of organic acids, mainly citrate, malate and succinate (Heerde and Radler I978), and depending on the different yield products there are differences in the way that cells have to cope with the redox balance. As yet, however, the nitrogen source strongly influences cellular metabolism and product formation in S. cerevisiae, especially owing to redox contour-balancing under anaerobic conditions (Albers et al. 1996). A high demand of NADPH is required for glutamate formation when cells are using ammonium as the only nitrogen source. In contrast, when glutamate is used as a nitrogen source the reduced synthesis of 2-oxoglutarate from glucose causes fewer reducing equivalents to be formed, which reduces the glycerol yield and hence increases the ethanol yield (Albers et al. I996; Camarasa et al. 2003).

The regulatory pattern of malic enzyme (encoded by $M A E 1$ ) suggests the specific physiological function in the provision of intramitochondrial NADPH or pyruvate in anaerobic metabolism; however, this enzyme does not have a strict function in redox metabolism, since null mutant in $M A E 1$ shows identical glycerol production under anaerobic conditions (Boles et al. I998).

\subsection{Yeast Growth Ability under Anaerobic Conditions: Is There a Common Prerequirement?}

Of the 678 recognized species, around $60 \%$ are considered to be fermentative on the basis of taxonomic tests such as gas production in Durham tubes (Barnett et al. 2000). However, this number is even higher since, under certain conditions, some of those species considered as nonfermentative are also able to ferment glucose (van Dijken et al. I986; Visser et al. I990). While the ability to ferment glucose under oxygen limitation turns out to be a common feature of the different yeast species, apparently the capability of growth under anaerobic conditions is not widespread among these microorganisms (Visser et al. I990). In fact, only very few yeast species are capable of fast growth under those conditions and $S$. cerevisiae stands out as the yeast generally acknowledged as a facultative anaerobe (Verduyn et al. I990; Visser et al. I990). It is commonly accepted that facultative anaerobes have the ability to grow both under aerobic and anaerobic conditions using, respectively, molecular oxygen or another compound as the final electron acceptor of the reducing equivalents that overflow from anabolic processes. Owing to the fact that anaerobic growth is associated with a low energy yield compared with that observed under complete oxidative processes, these microorganisms display two common characteristics: 
(I) the rate of sugar-substrate consumption is higher under anaerobic processes than that observed under aerobic conditions and (2) oxygen is used as a preferential source of the final electron acceptor.

As clearly pointed out by Lagunas (1979, I98I, I986), S. cerevisiae can roughly meet the previously described criteria for the reasons given hereafter. Even though $S$. cerevisiae is capable of rapid anaerobic growth it requires an external supply of sterols and fatty acids (Verduyn et al. 1990). The biosynthetic pathways involved in the production of these compounds, essential for membrane turnover, require molecular oxygen (Andreasen and Stier 1953, I954). Besides the expected benefits that would be produced from a shift from an anaerobic (where energy is the limiting factor) to an aerobic environment (with the putative ability to produce I.4-5.4 times more ATP per mole of sugar) they are almost irrelevant in $S$. cerevisiae (Lagunas I979, I986, and references therein; Rodrigues et al. 200 I). It should be stressed that in this species only around 5-10\% of glucose, maltose or fructose is not metabolized via alcoholic fermentation under aerobic batch growth. The ratios between the flux of those sugar consumptions under aerobic and anaerobic conditions are, respectively, I.05, 0.90 and I.08 (Lagunas I979). Ultimately, a rate value close to I means that no Pasteur effect occurs for the metabolism of the sugars described before.

Nevertheless, the biosynthetic oxygen requirements of facultative-fermentative yeasts are extremely small. Therefore, for reliable and meaningful identification of yeast species with the ability to grow under strictly anaerobic conditions special precautions (e.g., use of oxygen-resistant tubing and ultrapure nitrogen gas for sparking) are needed during the setup process of growth to minimize oxygen entry to the extent that these small oxygen requirements become apparent (Visser et al. I990; Rodrigues et al. 200I). Albeit, in some cases the requirements of molecular oxygen may be substantial, whereas, for instance, an insignificant leakage of oxygen between 0.3 and $6 \mathrm{mmol}$ of $\mathrm{O}_{2} \mathrm{~h}^{-1}$, corresponding to normal strict anaerobic conditions, is sufficient to allow the yeasts Zygosaccharomyces bailii and C. utilis to grow (Rodrigues et al. 200I). In spite of the linearity of the growth kinetics characteristics of oxygen limitation, $C$. utilis requires I.5-fold more time than $Z$. bailii to finish cell proliferation, highlighting the differences in oxygen demand of both species for growth. This raises the general question, what are the further limitations that explain the anaerobic growth incapability of "non-Saccharomyces" yeasts?

During the last few years, different research groups have raised several hypotheses, most of them related to the inability to fulfill the biosynthetic requirements under anaerobic conditions. Ergosterol and unsaturated fatty acids were shown to be essential for S. cerevisiae and S. kluyveri (Moller et al. 200I). Strengthening the specific requirements of $S$. cerevisiae for ergosterol and unsaturated fatty acids, (Wilcox et al. (2002) showed that the ATP-binding transporters Aus ip and Pdri Ip, associated with sterol uptake, are essential for anaerobic growth. However, it seems that Schizosaccharomyces japonicus does not require ergosterol for growth (Bulder 197I). On the other hand, the discovery by Nagy et al. (1992) that $S$. cerevisiae has one dihydroorotate dehydrogenase (DHODase) (encoded by the gene URA1), which catalyzes the single redox reaction converting dihidroorotate into orotate, not dependent on the functionality of the respiratory chain, opened a new line of research into the ability of yeasts to grow under anaerobic conditions. In this line, it was possible 
to turn the oxygen-dependent yeast Pichia stipitis into an anaerobic culturable yeast just by expressing the URAI gene from $S$. cerevisiae (Shi and Jeffries I998). More recently, it was experimentally shown that the genome of $S$. kluyveri also encodes a DHODase, functionally equivalent to that of $S$. cerevisiae (Gojkovic et al. 2004). The nice study of Gojkovic et al. (2004) illustrated that while aerobic yeasts, such as $S$. pombe and $C$. albicans contain just a mitochondrial DHODase, with a quininetype electron acceptor, the facultative anaerobes yeasts, such as $S$. cerevisiae, $S$. bayanus and $S$. castellii contain a cytosolic DHODase, with a fumarate-type electron acceptor, whereas $S$. kluyveri contains both. This study pointed to horizontal gene transfer from bacteria to the progenitor of these lineages, and raised the hypothesis that a remodeling of the biochemical pathways during yeast diversification took place, allowing the progressive reduction of oxygen requirements for growth. As yet, however, the capability of $Z$. bailii to grow under anaerobic conditions in yeast-peptone-glucose opposed to defined media is still unexplained since it is not due to uracil auxotrophy (Rodrigues et al. 200I). The involvement of additional biosynthetic requirements may perhaps be considered in other cases.

Another even more generalized limitation to anaerobic growth may be related to either/both a sufficiently high rate of ATP formation of a given substrate or/and an impaired mitochondrial ADP/ATP translocation, as well as constraints on redox metabolism (van Dijken and Scheffers I986; Verduyn et al. I990; Visser et al. I994; Trezeguet et al. 1999). Under anaerobic conditions and in contrast to aerobic conditions, the TCA cycle has only an anabolic function and the cell growth is completely dependent on ethanol formation for the provision of energy. In theory it is conceivable that, for certain yeast species, the glycolytic flux is not sufficient to drive the free energy for cellular processes. Verduyn et al. (I992) showed that the $S$. cerevisiae fermentation rate limits the supply of ATP for maintenance purposes above that required for anabolic reactions, to a value of approximately $\mathrm{I} 7 \mathrm{mmol}$ ATP Eq g ${ }^{-1} \mathrm{~h}^{-1}$. However, when the maintenance requirement increases to a threshold value (e.g., triggered by the uncoupling effect of weak carboxylic acids or by the decrease of the $\mathrm{pH}$ of the culture medium below 2.8), glycolytic flux can no longer provide further ATP, leading to cytosolic acidification and subsequently cell death (Verduyn et al. I990). It is to be stressed that the previous considerations are not related to the well-known Custers effect on yeast which is characterized by fermentation inhibition upon the transition to an anaerobic environment. In Torulaspora delbrueckii, it seems that the increase of glycolytic flux under anaerobic conditions is not sufficient to cope with growth demands (Hanl et al. 2004). On the other hand, an impairment of mitochondrial ADP/ATP translocation may lead to growth arrest in the absence of oxygen. In S. cerevisiae it was shown that the blockage of ADP/ATP translocators by bongkrekic acid induced a reduction of approximately $50 \%$ in the growth rate under anaerobic conditions (Visser et al. 1994). This dependence was further established by the inability of $S$. cerevisiae null mutant in $A A C$ genes ( $A A C 1, A A C 2, A A C 3$; coding ADP/ATP translocators) to grow under those conditions. One should emphasize that the same authors showed that this triple mutant was still able to grow on fermentable sources in the presence of oxygen and that $A A C 3$ was induced under anaerobic conditions (Kolarov et al. I990; Lawson et al. I990; Drgon et al. I99I). Furthermore, in S. cerevisiae batch cultivations, the 
surplus of reducing equivalents formed in anabolic reactions has to be balanced by the formation of glycerol estimated in approximately $5 \%$ of glucose breakdown. In this species, the null mutant in glycerol 3-phosphate dehydrogenase is incapable of growth under anaerobic conditions, and the simple deletion of the GPDI gene leads to a decrease of 5 times the growth rate (Nissen et al. 2000). More surprisingly, the same authors showed that the expression of a cytoplasmic transhydrogenase from Azotobacter vinelandii could not rescue the phenotype, showing that the $\mathrm{NAD}^{+}$pool that limited growth was lower than the threshold value favoring the transhydrogenase reaction. Therefore, an impaired redox metabolism may, in some cases, be behind the inability to grow anaerobically.

From everything discussed here, the hitherto unexplained inability of most yeast species to grow under anaerobic conditions is still an unsolved puzzle. Scientific efforts must, therefore, focus on an integrated approach keeping the essential strait balance between the two complementary approaches - the yeast physiology and the yeast molecular biology - and thus driving the expected increase in knowledge of yeast performance.

\section{Acknowledgment}

The authors thank Agostinho Almeida for critical English revision of the text.

\section{References}

Albers E, Larsson C, Liden G, Niklasson C, Gustafsson L (I996) Influence of the nitrogen source on Saccharomyces cerevisiae anaerobic growth and product formation. Appl Environ Microbiol 62:3I87-3I95

Andreasen AA, Stier TJB (I953) Anaerobic nutrition of Saccharomyce cerevisiae. I. Ergosterol requirement for growth in a defined medium. J Cell Comp Physiol 4I:23-36

Andreasen AA, Stier TJB (I954) Anaerobic nutrition of Saccharomyces cerevisiae. II. Unsaturated fatty acid requirement for growth in a defined medium. J Cell Comp Physiol 43:27I-28I

Ansell R, Granath K, Hohmann S, Thevelein JM, Adler L (I997) The two isoenzymes for yeast $\mathrm{NAD}^{+}$-dependent glycerol 3-phosphate dehydrogenase encoded by GPDI and GPD2 have distinct roles in osmoadaptation and redox regulation. EMBO J I6:2 I 79-2 I 87

Arikawa Y, Enomoto K, Muratsubakin H, Okazaki M (I998) Soluble fumarate reductase isoenzymes from Saccharomyces cerevisiae are required for anaerobic growth. FEMS Microbiol Lett I65: I I I-I I6

Atzpodien W, Gancedo JM, Duntze W, Holzer H ( I968) Isoenzymes of malate dehydrogenase in Saccharomyces cerevisiae. Eur J Biochem 7:58-62

Bakker BM, Bro C, Kotter P, Luttik MA, van Dijken JP, Pronk JT (2000) The mitochondrial alcohol dehydrogenase Adh3p is involved in a redox shuttle in Saccharomyces cerevisiae. J Bacteriol I 82:4730-4737

Bakker BM, Overkamp KM, van Maris AJ, Kotter P, Luttik MA, van Dijken JP, Pronk JT (200 I) Stoichiometry and compartmentation of NADH metabolism in Saccharomyces cerevisiae. FEMS Microbiol Rev 25: I 5-37

Barnett J, Payne R, Yarrow D (2000) Yeasts: characteristics and identification, 3rd edn. Cambridge University Press, Cambridge, UK

Beauvoit B, Rigoulet M, Bunoust O, Raffard G, Canioni P, Guerin B (I993) Interactions between glucose metabolism and oxidative phosphorylations on respiratory-competent Saccharomyces cerevisiae cells. Eur J Biochem 2I4:I63-I 72 
Bjorkqvist S, Ansell R, Adler L, Liden G (1997) Physiological response to anaerobicity of glycerol-3-phosphate dehydrogenase mutants of Saccharomyces cerevisiae. Appl Environ Microbiol 63: I 28-I32

Blank LM, Sauer U (2004) TCA cycle activity in Saccharomyces cerevisiae is a function of the environmentally determined specific growth and glucose uptake rates. Microbiology I 50: I085-I093

Blomberg A, Larsson C, Gustafsson L (I988) Microcalorimetric monitoring of growth of Saccharomyces cerevisiae: osmotolerance in relation to physiological state. $\mathrm{J}$ Bacteriol I $70: 4562-4568$

Boles E, Hollenberg CP (I997) The molecular genetics of hexose transport in yeasts. FEMS Microbiol Rev 2I:85-I I I

Boles E, de Jong-Gubbels P, Pronk JT (I998) Identification and characterization of MAEI, the Saccharomyces cerevisiae structural gene encoding mitochondrial malic enzyme. J Bacteriol I 80:2875-2882

Bruinenberg PM (I986) The NADP(H) redox couple in yeast metabolism. Antonie van Leeuwenhoek 52:4I I-429

Bruinenberg PM, van Dijken JP, Scheffers WA (I983) An enzymic analysis of NADPH production and consumption in Candida utilis. J Gen Microbiol I 29:965-97I

Bulder CJ (197I) Anaerobic growth, ergosterol content and sensitivity to a polyene antibiotic, of the yeast Schizosaccharomyces japonicus. Antonie van Leeuwenhoek 37:353-358

Bunoust O, Devin A, Averet N, Camougrand N, Rigoulet M (2005) Competition of electrons to enter the respiratory chain: a new regulatory mechanism of oxidative metabolism in Saccharomyces cerevisiae. J Biol Chem 280:3407-34I3

Camarasa C, Grivet JP, Dequin S (2003) Investigation by I3C-NMR and tricarboxylic acid (TCA) deletion mutant analysis of pathways for succinate formation in Saccharomyces cerevisiae during anaerobic fermentation. Microbiology I49:2669-2678

Ciriacy M ( I979) Isolation and characterization of further cis- and trans-acting regulatory elements involved in the synthesis of glucose-repressible alcohol dehydrogenase (ADHII) in Saccharomyces cerevisiae. Mol Gen Genet I 76:427-43 I

De Deken RH (I966) The Crabtree effect: a regulatory system in yeast. J Gen Microbiol 44: I49-I 56

De Jong-Gubbels P, Bauer J, Niederberger P, Stuckrath I, Kotter P, van Dijken JP, Pronk JT (I998) Physiological characterisation of a pyruvate-carboxylase-negative Saccharomyces cerevisiae mutant in batch and chemostat cultures. Antonie van Leeuwenhoek 74:253-263

De Vries S, Grivell LA (I988) Purification and characterization of a rotenone-insensitive NADH:Q6 oxidoreductase from mitochondria of Saccharomyces cerevisiae. Eur J Biochem I $76: 377-384$

De Vries S, Marres CA (I987) The mitochondrial respiratory chain of yeast. Structure and biosynthesis and the role in cellular metabolism. Biochim Biophys Acta 895:205-239

Dejean L, Beauvoit B, Guerin B, Rigoulet M (2000) Growth of the yeast Saccharomyces cerevisiae on a non-fermentable substrate: control of energetic yield by the amount of mitochondria. Biochim Biophys Acta I457:45-56

DeRisi JL, Iyer VR, Brown PO (I997) Exploring the metabolic and genetic control of gene expression on a genomic scale. Science 278:680-686

Drgon T, Sabova L, Nelson N, Kolarov J (I99I) ADP/ATP translocator is essential only for anaerobic growth of yeast Saccharomyces cerevisiae. FEBS Lett 289: I 59-I 62

Enomoto K, Arikawa Y, Muratsubaki H (2002) Physiological role of soluble fumarate reductase in redox balancing during anaerobiosis in Saccharomyces cerevisiae. FEMS Microbiol Lett 2I 5:103-I08 
Eriksson P, Andre L, Ansell R, Blomberg A, Adler L (I995) Cloning and characterization of GPD2, a second gene encoding sn-glycerol 3-phosphate dehydrogenase (NAD+) in Saccharomyces cerevisiae, and its comparison with GPDI. Mol Microbiol I 7:95-I07

Flikweert MT, van der Zanden L, Janssen WM, Steensma HY, van Dijken JP, Pronk JT (I996) Pyruvate decarboxylase: an indispensable enzyme for growth of Saccharomyces cerevisiae on glucose. Yeast I2:247-257

Flores CL, Rodriguez C, Petit T, Gancedo C (2000) Carbohydrate and energy-yielding metabolism in non-conventional yeasts. FEMS Microbiol Rev 24:507-529

Forsberg H, Ljungdahl PO (200I) Sensors of extracellular nutrients in Saccharomyces cerevisiae. Curr Genet 40:9I-109

Fukuhara H (2003) The Kluyver effect revisited. FEMS Yeast Res 3:327-33I

Gancedo C, Serrano R (1989) Energy yielding metabolism. In: Rose AH, Harrison JS (eds) The yeasts. Academic, London, pp 205-259

Gojkovic Z, Knecht W, Zameitat E, Warneboldt J, Coutelis JB, Pynyaha Y, Neuveglise C, Moller K, Loffler M, Piskur J (2004) Horizontal gene transfer promoted evolution of the ability to propagate under anaerobic conditions in yeasts. Mol Genet Genom 27I:387-393

Gombert AK, Moreira dos Santos M, Christensen B, Nielsen J (200I) Network identification and flux quantification in the central metabolism of Saccharomyces cerevisiae under different conditions of glucose repression. J Bacteriol I 83: I44I-I45I

Gonzaléz-Siso MI, Freire Picos MA, Cerdan ME (1996) Reoxidation of the NADPH produced by the pentose phosphate pathway is necessary for the utilization of glucose by Kluyveromyces lactis rag2 mutants. FEBS Lett 387:7-10

Gonzaléz-Siso MI, Freire-Picos MA, Ramil E, Gonzalez-Dominguez M, Rodriguez Torres A, Cerdan ME (2000) Respirofermentative metabolism in Kluyveromyces lactis: Insights and perspectives. Enzyme Microb Technol 26:699-705

Hanl L, Sommer P, Arneborg N (2004) The effect of decreasing oxygen feed rates on growth and metabolism of Torulaspora delbrueckii. Appl Microbiol Biotechnol 67:I I3-1 I 8

Heerde E, Radler F (1978) Metabolism of the anaerobic formation of succinic acid by Saccharomyces cerevisiae. Arch Microbiol I I 7:269-276

Jeffries TW, Jin YS (2004) Metabolic engineering for improved fermentation of pentoses by yeasts. Appl Microbiol Biotechnol 63:495-509

Kappeli O (1986) Regulation of carbon metabolism in Saccharomyces cerevisiae and related yeasts. Adv Microb Physiol 28:18I-203

Kiers J, Zeeman AM, Luttik M, Thiele C, Castrillo JI, Steensma HY, van Dijken JP, Pronk JT (I998) Regulation of alcoholic fermentation in batch and chemostat cultures of Kluyveromyces lactis CBS 2359. Yeast I4:459-469

Kispal G, Cseko J, Alkonyi I, Sandor A (I99I) Isolation and characterization of carnitine acetyltransferase from S. cerevisiae. Biochim Biophys Acta 1085:21 7-222

Kolarov J, Kolarova N, Nelson N (I990) A third ADP/ATP translocator gene in yeast. J Biol Chem 265:I27I I-I27I6

Kruckeberg AL, Dickinson JR (2004) Carbon metabolism. In: Dickinson JR, Schweizer M (eds) The metabolism and molecular physiology of Saccharomyces cerevisiae. CRC, London, pp 42-I03

Lagunas R (1979) Energetic irrelevance of aerobiosis for S. cerevisiae growing on sugars. Mol Cell Biochem 27:139-I46

Lagunas R (198I) Is Saccharomyces cerevisiae a typical facultative anaerobe? Trends Biochem Sci 6:20I-202

Lagunas R (1986) Misconceptions about the energy metabolism of Saccharomyces cerevisiae. Yeast 2:22I-228

Lagunas R (1993) Sugar transport in Saccharomyces cerevisiae. FEMS Microbiol Rev I0:229-242 
Larsson K, Ansell R, Eriksson P, Adler L (I993) A gene encoding sn-glycerol 3-phosphate dehydrogenase (NAD+) complements an osmosensitive mutant of Saccharomyces cerevisiae. Mol Microbiol Io: I Io I-I I I

Larsson K, Bohl F, Sjostrom I, Akhtar N, Strand D, Mechler BM, Grabowski R, Adler L (I998) The Saccharomyces cerevisiae SOPI and SOP2 genes, which act in cation homeostasis, can be functionally substituted by the Drosophila lethal(2)giant larvae tumor suppressor gene. J Biol Chem 273:336I0-336I8

Lawson JE, Gawaz M, Klingenberg M, Douglas MG (I990) Structure-function studies of adenine nucleotide transport in mitochondria. I. Construction and genetic analysis of yeast mutants encoding the ADP/ATP carrier protein of mitochondria. J Biol Chem 265: I4I I95-I420 I

Luttik MA, Overkamp KM, Kotter P, de Vries S, van Dijken JP, Pronk JT (I998) The Saccharomyces cerevisiae NDEI and NDE2 genes encode separate mitochondrial NADH dehydrogenases catalyzing the oxidation of cytosolic NADH. J Biol Chem 273:24529-24534

Marres CA, de Vries S, Grivell LA (I99I) Isolation and inactivation of the nuclear gene encoding the rotenone-insensitive internal NADH: ubiquinone oxidoreductase of mitochondria from Saccharomyces cerevisiae. Eur J Biochem I95:857-862. Erratum in: Eur J Biochem (I99I) 200:8I 2

McAlister-Henn L, Small WC (I997) Molecular genetics of yeast TCA cycle isozymes. Prog Nucleic Acid Res Mol Biol 57:3 I 7-339

McCammon MT, Epstein CB, Przybyla-Zawislak B, McAlister-Henn L, Butow RA (2003) Global transcription analysis of Krebs tricarboxylic acid cycle mutants reveals an alternating pattern of gene expression and effects on hypoxic and oxidative genes. Mol Biol Cell I4:958-972

Meaden PG, Dickinson FM, Mifsud A, Tessier W, Westwater J, Bussey H, Midgley M (I997) The ALD6 gene of Saccharomyces cerevisiae encodes a cytosolic, $\mathrm{Mg}(2+)$-activated acetaldehyde dehydrogenase. Yeast I3:I3I9-I327

Minard KI, Jennings GT, Loftus TM, Xuan D, McAlister-Henn L (I998) Sources of NADPH and expression of mammalian $\mathrm{NADP}^{+}$-specific isocitrate dehydrogenases in Saccharomyces cerevisiae. J Biol Chem 273:3I486-3I493

Moller IM, Rasmusson AG, Fredlund KM (I993) NAD(P)H-ubiquinone oxidoreductases in plant mitochondria. J Bioenerg Biomembr 25:377-384

Moller K, Olsson L, Piskur J (200I) Ability for anaerobic growth is not sufficient for development of the petite phenotype in Saccharomyces kluyveri. J Bacteriol I 83:2485-2489

Nagy M, Lacroute F, Thomas D (I992) Divergent evolution of pyrimidine biosynthesis between anaerobic and aerobic yeasts. Proc Natl Acad Sci USA 89:8966-8970

Nissen TL, Schulze U, Nielsen J, Villadsen J (I 997) Flux distributions in anaerobic, glucoselimited continuous cultures of Saccharomyces cerevisiae. Microbiology I43:203-2 I 8

Nissen TL, Hamann CW, Kielland-Brandt MC, Nielsen J, Villadsen J (2000) Anaerobic and aerobic batch cultivations of Sacharomyces cerevisiae mutants impaired in glycerol synthesis. Yeast I 6:463-474

Norbeck J, Pahlman AK, Akhtar N, Blomberg A, Adler L (I996) Purification and characterization of two isoenzymes of DL-glycerol-3-phosphatase from Saccharomyces cerevisiae. Identification of the corresponding GPPI and GPP2 genes and evidence for osmotic regulation of Gpp2p expression by the osmosensing mitogen-activated protein kinase signal transduction pathway. J Biol Chem 27I:I3875-I388 I

Nosek J, Fukuhara H (I994) NADH dehydrogenase subunit genes in the mitochondrial DNA of yeasts. J Bacteriol I 76:5622-5630

Nunez de Castro I, Ugarte M, Cano A, Mayor F (1970) Effect of glucose, galactose, and different nitrogen-sources on the activity of yeast glutamate dehydrogenase (NAD and 
NADP-linked) from normal strain and impaired respiration mutant. Eur J Biochem I 6:567-570

Overkamp KM, Bakker BM, Kotter P, van Tuijl A, de Vries S, van Dijken JP, Pronk JT (2000) In vivo analysis of the mechanisms for oxidation of cytosolic NADH by Saccharomyces cerevisiae mitochondria. J Bacteriol I 82:2823-2830

Pahlman AK, Granath K, Ansell R, Hohmann S, Adler L (200I) The yeast glycerol 3-phosphatases Gppip and Gpp2p are required for glycerol biosynthesis and differentially involved in the cellular responses to osmotic, anaerobic, and oxidative stress. J Biol Chem 276:3555-3563

Petrik M, Kappeli O, Fiechter, A (1983) An expanded concept for the glucose effect in the yeast Saccharomyces uvarum: involvment of short- and long-term regulation. J Gen Microbiol I 29:43-49

Pronk JT, Yde Steensma H, van Dijken JP (I996) Pyruvate metabolism in Saccharomyces cerevisiae. Yeast I 2:I607-I633

Przybyla-Zawislak B, Gadde DM, Ducharme K, McCammon MT (I999) Genetic and biochemical interactions involving tricarboxylic acid cycle (TCA) function using a collection of mutants defective in all TCA cycle genes. Genetics I 52:I53-I66

Remize F, Andrieu E, Dequin S (2000) Engineering of the pyruvate dehydrogenase bypass in Saccharomyces cerevisiae: role of the cytosolic $\mathrm{Mg}(2+)$ and mitochondrial $\mathrm{K}(+)$ acetaldehyde dehydrogenases Ald6 $\mathrm{p}$ and Ald $4 \mathrm{p}$ in acetate formation during alcoholic fermentation. Appl Environ Microbiol 66:3I 5I-3I59

Richard P (2003) The rhythm of yeast. FEMS Microbiol Rev 27:547-557

Rigoulet M, Aguilaniu H, Averet N, Bunoust O, Camougrand N, Grandier-Vazeille X, Larsson C, Pahlman IL, Manon S, Gustafsson L (2004) Organization and regulation of the cytosolic NADH metabolism in the yeast Saccharomyces cerevisiae. Mol Cell Biochem 256/257:73-8I

Rodrigues F, Corte-Real M, Leao C, van Dijken JP, Pronk JT (200I) Oxygen requirements of the food spoilage yeast $Z$ ygosaccharomyces bailii in synthetic and complex media. Appl Environ Microbiol 63:2I 23-2I 28

Rustin P, Bourgeron T, Parfait B, Chretien D, Munnich A, Rotig A (1997) Inborn errors of the Krebs cycle: a group of unusual mitochondrial diseases in human. Biochim Biophys Acta I36I:I85-I97

Saint-Prix F, Bonquist L, Dequin S (2004) Functional analysis of the ALD gene family of Saccharomyces cerevisiae during anaerobic growth on glucose: the NADP ${ }^{+}$-dependent Ald6p and Ald 5 p isoforms play a major role in acetate formation. Microbiology I 50:2209-2220

Sass E, Blachinsky E, Karniely S, Pines O (200I) Mitochondrial and cytosolic isoforms of yeast fumarase are derivatives of a single translation product and have identical amino termini. J Biol Chem 276:46 I I I-46I I 7

Schagger H, Pfeiffer K (2000) Supercomplexes in the respiratory chains of yeast and mammalian mitochondria. EMBO J I9:I777-I 783

Sheffers WA (1966) Stimulation of fermentation by acetoin and oxygen. Nature 210:533-534

Shi NQ, Jeffries TW (1998) Anaerobic growth and improved fermentation of Pichia stipitis bearing a URA gene from Saccharomyces cerevisiae. Appl Microbiol Biotechnol 50:339-345

Small WC, McAlister-Henn L (1998) Identification of a cytosolically directed NADH dehydrogenase in mitochondria of Saccharomyces cerevisiae. J Bacteriol I80:405I-4055

Sprague GF, Cronan JE (1977) Isolation and characterization of Saccharomyces cerevisiae mutants defective in glycerol catabolism. J Bacteriol I29:I335-I342

Steensma HY (1997) From pyruvate to acetyl-CoA and oxaloacetate. In: Zimmermann FK, Entian KD (eds) Yeast sugar metabolism - biochemistry, genetics, biotechnology, and applications. Technomic, Lancaster, pp 339-357 
Steffan JS, McAlister-Henn L (I992) Isolation and characterization of the yeast gene encoding the $\mathrm{MDH}_{3}$ isozyme of malate dehydrogenase. J Biol Chem 267:24708-247I 5

Swanson WH, Clifton CE (I948) Growth and assimilation in cultures of Saccharomyces cerevisiae. J Bacteriol 56: I I 5-I 24

Ter Linde JJ, Liang H, Davis RW, Steensma HY, van Dijken JP, Pronk JT (I999) Genomewide transcriptional analysis of aerobic and anaerobic chemostat cultures of Saccharomyces cerevisiae. J Bacteriol I8I:7409-74I3

Tessier WD, Meaden PG, Dickinson FM, Midgley M (I998) Identification and disruption of the gene encoding the $\mathrm{K}(+)$-activated acetaldehyde dehydrogenase of Saccharomyces cerevisiae. FEMS Microbiol Lett I 64:29-34

Trezeguet V, Zeman I, David C, Lauquin GJM, Kolarov J (I999) Expression of the ADP/ATP carrier encoding genes in aerobic yeasts; phenotype of an ADP/ATP carrier deletion mutant of Schizosaccharomyces pombe. Biochim Biophys Acta I4I0:229-236

Van den Berg MA, Steensma HY (1995) ACS2, a Saccharomyces cerevisiae gene encoding acetyl-coenzyme A synthetase, essential for growth on glucose. Eur J Biochem 23 I:704-7I3

Van Dijken JP, Scheffers WA (I986) Redox balances in the metabolism of sugars by yeasts. FEMS Microbiol Rev 32:199-224

Van Dijken JP, van den Bosch E, Hermans JJ, de Miranda LR, Scheffers WA (I986) Alcoholic fermentation by 'non-fermentative' yeasts. Yeast 2: I 23-I 27

Van Urk H, Bruinenberg PM, Veenhuis M, Scheffers WA, Van Dijken JP (I989) Respiratory capacities of mitochondria of Saccharomyces cerevisiae CBS 8066 and Candida utilis CBS 62I grown under glucose limitation. Antonie van Leeuwenhoek 56:2 I I-220

Vanlerberghe GC, McIntosh L ( I 997) Alternative oxidase: from gene to function. Annu Rev Plant Physiol Plant Mol Biol 48:703-734

Verduyn C (I99I) Physiology of yeasts in relation to biomass yields. Antonie van Leeuwenhoek 60:325-353

Verduyn C, Postma E, Scheffers WA, van Dijken JP (I990) Physiology of Saccharomyces cerevisiae in anaerobic glucose-limited chemostat cultures. J Gen Microbiol 59:49-63

Verduyn C, Postma E, Scheffers WA, van Dijken JP (I 992) Effect of benzoic acid on metabolic fluxes in yeasts: a continuous culture study on the regulation of respiration and alcoholic fermentation. Yeast 8:50I-5I 7

Visser W, Batenburg-van der Vegte W, Scheffers WA, van Dijken JP (I990) Oxygen requirements of yeasts. Appl Environ Microbiol 56:3785-3792

Visser W, van der Baan AA, Batenburg-van der Vegte W, Scheffers WA, Kramer R, van Dijken JP (I994) Involvement of mitochondria in the assimilatory metabolism of anaerobic Saccharomyces cerevisiae cultures. Microbiology I40:3039-3046

Von Jagow G, Klingenberg M (1970) Pathways of hydrogen in mitochondria of Saccharomyces carlsbergensis. Eur J Biochem I2:583-592

Wang X, Mann CJ, Bai Y, Ni L, Weiner H (I998) Molecular cloning, characterization, and potential roles of cytosolic and mitochondrial aldehyde dehydrogenases in ethanol metabolism in Saccharomyces cerevisiae. J Bacteriol I80:822-830

Wijsman MR, van Dijken JP, van Kleeff BH, Scheffers, WA (I984) Inhibition of fermentation and growth in batch cultures of the yeast Brettanomyces intermedius upon a shift from aerobic to anaerobic conditions (Custer effect). Antonie van Leeuwenhoek 50: I 83-I92

Wilcox LJ, Balderes DA, Wharton B, Tinkelenberg AH, Rao G, Sturley SL (2002) Transcriptional profiling identifies two members of the ATP-binding cassette transporter superfamily required for sterol uptake in yeast. J Biol Chem 277:32466-32472

Yudkoff M, Nelson D, Daikhin Y, Erecinska M (1994) Tricarboxylic acid cycle in rat brain synaptosomes. Fluxes and interactions with aspartate aminotransferase and malate/aspartate shuttle. J Biol Chem 269:274I4-27420 
